\title{
Postoperative back pain
}

\author{
SA Worthy, DL Richardson, D Lambert
}

A 71-year-old man presented with a four-week history of left-sided back and leg pain. Two and a half years previously he had had an aorto-bifemoral prosthetic graft replacement for an inflammatory aortic aneurysm which extended into both iliac arteries. He had a history of ischaemic heart disease and a strong family history of ischaemic heart disease.

On examination the only significant finding was marked wasting of the muscles of the left leg. Initial investigations demonstrated degenerative changes in the lumbar spine, to which his symptoms were originally attributed. The left-sided pain had persisted for three months when he was admitted to hospital with an exacerbation of the back pain and left leg pain. A contrastenhanced computed tomography (CT) scan was performed (figure 1).

Royal Victoria Infirmary, Queen Victoria Road, Newcastle upon Tyne NE1 4LP, UK

Department of Radiology

SA Worthy

DL Richardson

Department of

Surgery

D Lambert

Correspondence to Dr SA

Worthy, 50 Cornmoor Road,

Whickham NE16 4PU, UK

Accepted 16 October 1995
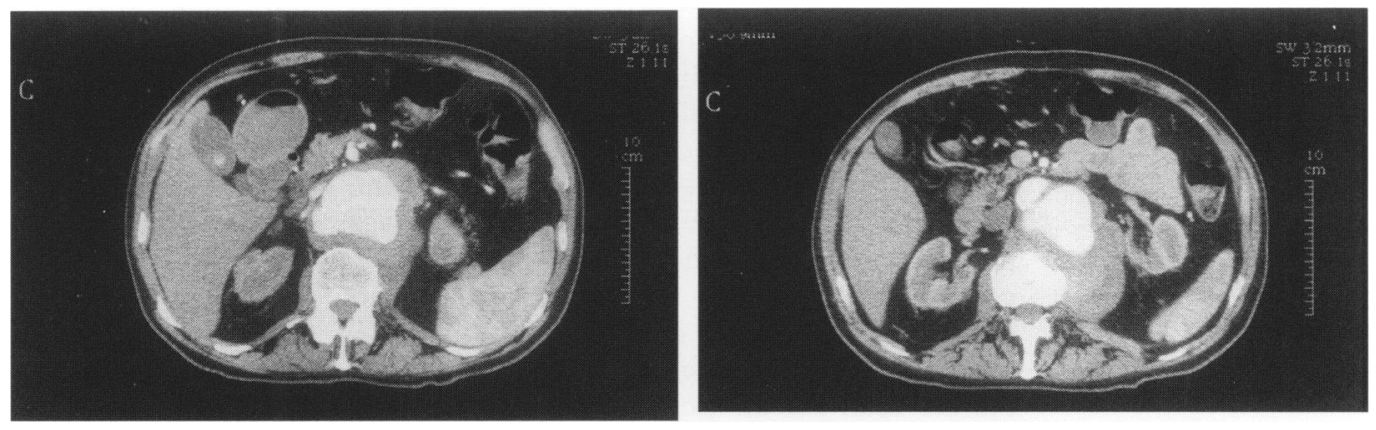

Figure 1 Contrast-enhanced CT scan of the abdomen

\section{Questions}

1 What is the most likely cause of the back pain?

2 Why did this patient have left leg pain and muscle wasting? 


\section{Answers}

\section{QUESTION}

The cause of the back pain demonstrated by the CT scan was a large false aneurysm arising just below the level of the superior mesenteric artery with an enhancing false lumen extending posteriorly and to the left where it extended into the psoas muscle. Erosion of the vertebral body by the peri-aortic tissue was shown. Further sections showed that the left renal artery was displaced anteriorly by peri-aortic soft tissue and the left kidney showed poor contrast excretion. The aorta at the coeliac axis level was also aneurysmal. A diagnosis of a para-anastomotic aortic false aneurysm was made and confirmed by intravenous digital subtraction angiography.

Figure 2 demonstrates the appearance of the aneurysm on a three-dimensional reconstruction image of the CT scan with the superior mesenteric artery (white arrow) and right renal artery (black arrow) marked. Operative findings demonstrated dilatation of the aorta proximal to the proximal graft anastomosis and a para-anastomotic false aneurysm arising posteriorly at that level and extending into the left psoas. A further prosthetic graft was inserted proximal to the original graft and the coeliac axis, superior mesenteric artery and right renal artery were anastomosed to it.

The gallstone in the gallbladder shown on the CT scan was an incidental finding and was unlikely to account for the severity of the symptoms.

\section{QUESTION 2}

The left leg pain and muscle wasting were caused by extension of the para-anastomotic false aneurysm into the left psoas muscle resulting in compression of the lumbar plexus nerves which run in the posterior part of the psoas muscle, thus mimicking spinal pathology as the underlying cause.

\section{Discussion}

Prosthetic grafting of the abdominal aorta is commonly performed for aneurysmal or occlusive disease. Complicarions following aortoiliac reconstruction are infrequent and include thrombosis, aneurysm, infection and graft-

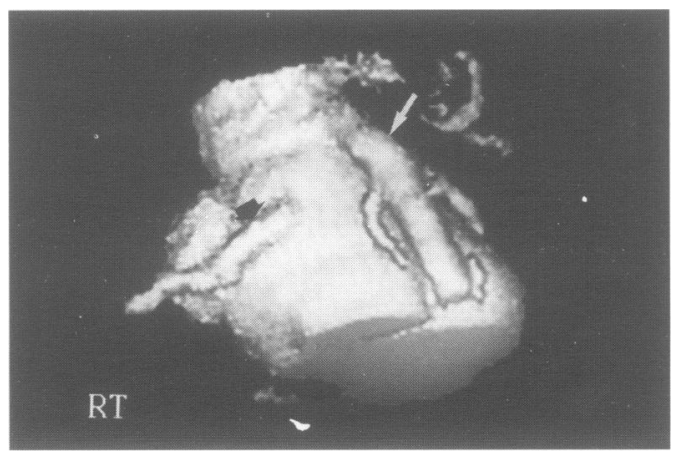

Figure 2 Three dimensional reconstruction of the aorta and its branches

\section{Learning points}

- degenerative changes on a lumbar spine radiograph are common in older patients and may not be the cause of back pain

- screening following aortic aneurysm repair may improve the outcome of patients who develop para-anastomotic aneurysms

- CT is a sensitive and accurate imaging method for detecting postoperative complications following aortic reconstruction

enteric fistula. ${ }^{1}$ Postoperative para-anastomotic aneurysm (PAA) formation is an uncommon but serious complication, the reported incidence being $0.2-15 \% .{ }^{2}$ PAA formation occurs more frequently in patients whose original pathology was aneurysmal rather than occlusive disease and it is more common at anastomoses in the femoral region than in the aorto-iliac region. ${ }^{3}$ The cause of PAA is probably multifactorial. A true aneurysm is contained within an intrinsic defect in the arterial wall. It may result either from progressive degeneration of the vessel wall following surgery or from failure to resect all the affected portion of the vessel at the time of surgery. A false aneurysm is not enclosed by arterial wall and results from a defect in the native arterial wall, the graft or a rupture at the suture line. Factors which may affect the development of false aneurysms include the type of suture material, type of graft material, method of anastomosis and presence of infection. Early PAA formation may occur when the early postoperative course is complicated.

PAA may be asymptomatic and detected fortuitously or may present with abdominal pain, back pain or claudication. PAAs tend to be detected late once they become symptomatic by which time they are large. In a series of 29 patients with PAA the average diameter at presentation was $7 \mathrm{~cm}^{2}$ In most centres there is no routine screening for the development of postoperative aneurysm formation, although both ultrasound and CT are sensitive and accurate methods of aneurysm detection..$^{3,4}$ Some centres have reported a high rate of accuracy with ultrasound. ${ }^{2} \mathrm{CT}$ is a rapid, sensitive, noninvasive method of demonstrating the postoperative complications following abdominal aortic reconstruction. It is able to demonstrate the size and extent of a PAA. PAAs progressively increase in size; rupture may occur at any stage in the postoperative course and has a poor survival. A PAA may also erode into bowel or cause ureteric obstruction. ${ }^{4}$ Early detection and elective repair is recommended. ${ }^{2}$ Adequate pre-operative work-up of patients with PAA is required to allow planning of the appropriate surgical procedure. A thoraco-abdominal approach is often needed to provide adequate exposure for a proximal PAA repair. Routine screening has been proposed to improve the outcome of patients who develop PAAs. ${ }^{2}$

This case illustrates the value of CT in the investigation of the patient's pain and in providing the true diagnosis. 
Final diagnosis

Para-anastomotic aortic false aneurysm with extension into the left psoas muscle and consequent compression of the lumbar plexus nerves.

1 Haiart DC, Callam MJ, Murie JA, Ruckley CV, Jenkins AM Reoperations for late complications following abdominal Reoperations for late complications following
aortic operations. $B r ₹$ Surg 1991; 78: 204-6.

2 aortic operations. Br $f$ Surg 1991; 78: 204-6. AB. Paraanastomotic aneurysms of the abdominal aorta. $f$ Vasc Surg 1993; 18: 424-31.
Keywords: aneurysm, aorta, computed tomography

3 Hilton S, Megibow AJ, Naidich DP, Bosniak MA. Computed tomography of the postoperative abdominal aorta Radiology 1982; 145: $403-7$.

4 Mark A, Moss AA, Lusby R, Kaiser JA. CT evaluation of abdominal aortic surgery. Radiology 1982; 145: 409-14.

\title{
Acute paralytic illness
}

\author{
D Kidd, H Manji, D Brown, RS Howard
}

Departments of Neurology, St Thomas'

Hospital, London SE1 7EH, UK

D Kidd

RS Howard

National Hospital for Neurology and Neurosurgery, Maida

Vale, London, UK

H Manji

RS Howard

Central Public Health Laboratory, Public

Health Laboratory

Service, London, UK

D Brown

Correspondence to Dr RS Howard at St Thomas' Hospita

Accepted 28 February 1996
A 21-year-old man presented with the sudden onset of weakness of the left leg, low back pain and tingling around the lower abdomen. On the following day he developed fever, severe generalised headache, photophobia, breathlessness and urinary hesitancy. One week later he developed dyspnoea, limb weakness, difficulty sitting up and had generalised paraesthesiae, particularly affecting his hands, feet and periorbital regions. He was admitted to hospital and at that time it was noted that he was unable to lift his leg off the bed and had severe difficulty in walking. Cerebrospinal fluid (CSF) protein was $0.2 \mathrm{~g} / 1$, white cell count $80 \mathrm{cells} / \mathrm{ml}$ ( $100 \%$ lymphocytes). A diagnosis of viral meningitis was made and he was treated with intravenous Acyclovir.

Over the course of the next six weeks the situation remained unchanged; his breathlessness continued, particularly lying flat, and there was weakness of the right arm and left leg. On examination, he walked with a left Trendelenberg gait and right shoulder droop. The cranial nerves were normal. In the upper limbs there was wasting of right triceps, spinati and deltoid. There was severe weakness of these muscles and moderate weakness of biceps, brachioradialis and triceps, and mild distal weakness. The left arm was normal. In the lower limbs there was wasting of the left quadriceps, with severe weakness of abduction, adduction and extension of the left hip and moderate weakness of knee flexion, ankle dorsiflexion, inversion and eversion. The left ankle reflex was absent and the plantar responses were flexor. Sensation was normal. Forced vital capacity was 6.21 erect and 4.91 lying; diaphragmatic screening confirmed evidence of mild diaphragmatic weakness.

It was noted that he had never received vaccination against polio in childhood. His son who was seven months old had received two doses of DPT and polio vaccinations, the second of which had been two weeks before the onset of his father's illness. He was in the habit of changing the child's nappy.

\section{Questions}

1 What is the most likely diagnosis?

2 What four further investigations should be performed?

3 What is the probable outcome? 